\title{
Collapse of Microfluidic Channels/Reservoirs in Thin, Soft Epidermal Devices
}

\author{
Yeguang Xue ${ }^{1}$, Daeshik Kang ${ }^{2,3}$, Yinji Ma ${ }^{1,4}$, Xue Feng ${ }^{4}$, John A. Rogers ${ }^{2}$ and Yonggang Huang ${ }^{1^{*}}$
}

${ }^{1}$ Department of Civil and Environmental Engineering, Mechanical Engineering, and Materials

Science and Engineering, Northwestern University, Evanston, IL 60208, USA

${ }^{2}$ Department of Materials Science and Engineering, Frederick Seitz Materials Research Laboratory, University of Illinois at Urbana-Champaign, Urbana, IL 61801, USA

${ }^{3}$ Department of Mechanical Engineering, Ajou University, Suwon, 443-749, Korea

${ }^{4}$ Department of Engineering Mechanics, Center for Mechanics and Materials, Tsinghua University, Beijing 10084, China

* To whom correspondence should be addressed. E-mail: y-huang@northwestern.edu

\begin{abstract}
Self-collapse is a common problem encountered in fabrication of thin, soft epidermal microfluidic devices, due to the adhesion between top and bottom covers. Analytic models are developed for collapse of both long microfluidic channels and circular microfluidic reservoirs, with their covers modelled as plane-strain beam and thin plate, respectively. The analysis shows that a single parameter, the normalized work of adhesion, which combines the effects of channel/reservoir geometry, work of adhesion and bending stiffness of top and bottom channel/reservoir covers, controls different collapse states (no collapse, meta stable collapse and stable collapse). The established models agree well with the experimental observations, and provide guidelines to avoid the problem of self-collapse in design of epidermal microfluidic devices.
\end{abstract}




\section{Introduction}

Wearable electronic devices enable real time, continuous monitoring that are essential to personalized health care [1, 2]. Although many devices reported in the literature are designed to track health status through measurement of physical characteristics (e.g. temperature, motion, strain, biopotential) [3-5], there is growing interest in the capture and biochemical analysis of biofluids (e.g. sweat) using these same types of platforms, as a route to complementary information of high clinic values [6-8]. A particularly notable system incorporates microfluidic networks and reservoirs in soft, elastomeric materials, capable of capturing and routing sweat for the purposes of storage and chemical analysis of key biomarkers (e.g., pH, concentration of biomarkers of interest) [9]. Low modulus elastomers and thin geometries provide mechanical compatibility with the skin, to reduce the probability of interface irritation and discomfort $[10,11]$. These same properties, however, result in systems that are vulnerable to structural instability, where self-collapse of the channels represents a common challenge. This sort of collapse results from adhesion between top and bottom covers of the soft channels/reservoirs. Careful mechanical design is required to avoid such issues.

This paper focuses on the criteria for collapse in microfluidic systems. The criteria are developed for microfluidic devices in dry environment because devices are most vulnerable to collapse in such condition. After filling with liquid, the inner pressure provides additional resistance to collapse and the work of adhesion in liquid-filled environment is also typically lower. The model accounts for the difference in bending stiffness of top and bottom covers, which is common among microfluidic epidermal devices because of additionally attached layers, 
such as the adhesive layer used to bond the device with skin and the electronics layer used for communication. The paper is structured as followed: Section 2 focuses on collapse of the long microfluidic channels used for routing biofluids, and section 3 focuses on collapse of the circular microfluidic reservoirs used for storage and biochemical analysis. The analytic models established are validated by experiments in section 4 .

\section{Collapse of Microfluidic Channels}

A 2D, plane-strain model is developed in this section for the long, microfluidic channels. These channels, either straight or serpentine along the length direction, have a rectangular crosssection with width $2 a$ and height $2 h$ (Fig. 1a). Figure 1 b shows the collapsed state with length $2 b$ (to be determined). Contact between the top and bottom covers of the channel reduces the surface energy by $-2 b \gamma[12,13]$, where $\gamma$ is the work of adhesion between the materials of the top and bottom covers. The total potential energy at the collapsed state is

$$
U_{\text {total }}=U_{\text {deformation }}-2 b \gamma
$$

The top and bottom covers are modelled as plane-strain beams because their thickness $t \ll a$ and they extend very long in the length direction. The two covers have different bending stiffness $D_{\text {top }}$ and $D_{\text {bottom }}$ for top and bottom covers, respectively. For channel cover composed of a single layer, the bending stiffness is given as $D=E t^{3} /\left[12\left(1-v^{2}\right)\right]$ in terms of Young's modulus $E$, Poisson's ratio $v$, and thickness $t$ of the cover. For inhomogeneous cover composed of multiple layers, the bending stiffness can be calculated using composite beam theory.

It can be proved that in collapsed state, the collapsed region always keeps flat, which corresponds to the energy minimum (see Appendix A). Thus, for the top cover, the deflection 
$w$ in the collapsed region $(|x| \leq b)$ is constant $-\delta$ to be determined. In the uncollapsed region, it satisfies

$$
\frac{\mathrm{d}^{4} w}{\mathrm{~d} x^{4}}=0 \quad(b<x<a)
$$

with the boundary conditions

$$
\left\{\begin{array}{c}
\left.w\right|_{x=a}=0 \\
\left.\frac{\mathrm{d} w}{\mathrm{~d} x}\right|_{x=a}=0 \\
\left.w\right|_{x=b}=-\delta \\
\left.\frac{\mathrm{d} w}{\mathrm{~d} x}\right|_{x=b}=0
\end{array} .\right.
$$

Its solution is

$$
w(x)=\left\{\begin{array}{cc}
-\delta & |x| \leq b \\
-\delta \frac{(a-|x|)^{2}(a-3 b+2|x|)}{(a-b)^{3}} & b<|x| \leq a
\end{array},\right.
$$

which gives the deformation energy in the top cover as

$$
U_{\text {deformation }}^{\text {top cover }}=\int_{-a}^{-b} \frac{D_{\text {top }}}{2}\left(\frac{\mathrm{d}^{2} w}{\mathrm{~d} x^{2}}\right)^{2} \mathrm{~d} x+\int_{b}^{a} \frac{D_{\text {top }}}{2}\left(\frac{\mathrm{d}^{2} w}{\mathrm{~d} x^{2}}\right)^{2} \mathrm{~d} x=\frac{12 D_{\text {top }} \delta^{2}}{(a-b)^{3}} .
$$

For the bottom cover, the deflection $w$ in the collapsed region $(|x| \leq b)$ is $2 h-\delta$, and the deformation energy can be calculated as

$$
U_{\text {deformation }}^{\text {bottom cover }}=\frac{12 D_{\text {bottom }}(2 h-\delta)^{2}}{(a-b)^{3}} .
$$

Substitution of Eqs. 5 and 6 into Eq. 1 gives the total potential energy

$$
U_{\text {total }}=\frac{12 D_{\text {top }} \delta^{2}}{(a-b)^{3}}+\frac{12 D_{\text {bottom }}(2 h-\delta)^{2}}{(a-b)^{3}}-2 b \gamma .
$$

The deflection of collapsed region of top cover $\delta$ is first determined by the minimization of total energy with respect to the deflection $\delta\left(\partial U_{\text {total }} / \partial \delta=0\right)$, which gives 


$$
\frac{\delta}{2 h}=\frac{D_{\text {bottom }}}{D_{\text {top }}+D_{\text {bottom }}}
$$

Substitution of the above into Eq. 7 gives the total energy expression with only collapse length $b$ to be determined.

$$
U_{\text {total }}=\frac{24 D_{\mathrm{eff}} h^{2}}{a^{3}}\left[\frac{1}{\left(1-\frac{b}{a}\right)^{3}}-\frac{\gamma a^{4}}{12 D_{\mathrm{eff}} h^{2}}\left(\frac{b}{a}\right)\right]
$$

where $D_{\text {eff }}$ is the effective bending stiffness defined as

$$
D_{\text {eff }}=\frac{2 D_{\text {top }} D_{\text {bottom }}}{D_{\text {bottom }}+D_{\text {top }}}
$$

In Eq. 9, the normalized total potential energy $U_{\text {total }} a^{3} /\left(D_{\text {eff }} h^{2}\right)$ depends only on the normalized collapse length $b / a$ and normalized work of adhesion,

$$
\bar{\gamma}_{\text {channel }}=\frac{\gamma a^{4}}{D_{\text {eff }} h^{2}}=\frac{\gamma\left(D_{\text {bottom }}+D_{\text {top }}\right) a^{4}}{2 D_{\text {top }} D_{\text {bottom }} h^{2}},
$$

which combines the effects of work of adhesion, bending stiffness of top and bottom covers, channel height and width. Figure 2 shows the normalized total potential energy $U_{\text {total }} a^{3} /\left(D_{\text {eff }} h^{2}\right)$ versus normalized collapse length $b / a$ for the normalized work of adhesion $\bar{\gamma}_{\text {channel }}=0,36,72,113.78$ and 200 . The collapse length $2 b_{\text {critical }}$ determined by minimizing the total potential energy $\left(\partial U_{\text {total }} / \partial b=0\right.$ and $\left.\partial^{2} U_{\text {total }} / \partial b^{2}>0\right)$ is also shown. For $\bar{\gamma}_{\text {channel }}<$ 36 , no solution exists for $b_{\text {critical }} / a$ because $U_{\text {total }}$ increases monotonically with $b / a$ and there is no minimum. Consequently, there is no self-collapse for weak adhesion $\bar{\gamma}_{\text {channel }}<36$. For $\bar{\gamma}_{\text {channel }}>36$, minimization of the total potential energy gives $b_{\text {critical }} / a$

$$
\frac{b_{\text {critical }}}{a}=1-\left(\frac{36}{\bar{\gamma}_{\text {channel }}}\right)^{\frac{1}{4}} \text { for } \bar{\gamma}_{\text {channel }}>36 .
$$


The normalized collapsed length $b_{\text {critical }} / a$ increases with the work of adhesion for $\bar{\gamma}_{\text {channel }}>36$ and approaches 1 (complete collapse). Substitution of Eq. 12 into Eq. 9 gives the minimum total potential energy $U_{\text {total }}^{\min }$

$$
U_{\text {total }}^{\min }=\frac{24 D_{\text {eff }} h^{2}}{a^{3}}\left[4\left(\frac{\bar{\gamma}_{\text {channel }}}{36}\right)^{\frac{3}{4}}-\frac{\bar{\gamma}_{\text {channel }}}{12}\right] .
$$

For intermediate adhesion $36<\bar{\gamma}_{\text {channel }} \leq 113.78, U_{\text {total }}^{\min }$ is above zero, the energy of uncollapsed state, such that the collapse is only meta stable, i.e., it may revert back to the uncollapsed state. For strong adhesion $\bar{\gamma}_{\text {channel }}>113.78, U_{\text {total }}^{\min }$ is below zero such that the collapsed state gives the lowest state of energy and therefore the collapse is stable.

In real microfluidic devices, if one cover is much stiffer than the other, e.g. $D_{\text {bottom }} \gg$ $D_{\text {top }}=D$, from Eq. 8 the deflection of top cover $\delta \approx 2 h$ and the problem reduces to one sided collapse studied in the adhesion of microstructures in MEMS devices[14-16]. From $\bar{\gamma}_{\text {channel }} \approx$ $\gamma a^{4} /\left(2 D h^{2}\right)$, and no collapse requires $\gamma a^{4} /\left(2 D h^{2}\right)<36$, which is consistent with the reported result[14].

\section{Collapse of Microfluidic Reservoirs}

An axisymmetric model is developed in this section for collapse of the circular microfluidic reservoir. Figure 3 shows the diagram of a microfluidic reservoir of radius $2 R$ and its collapsed state. The collapsed region is also circular due to axis-symmetry, with the radius $r$ to be determined. The total potential energy at the collapsed state is

$$
U_{\text {total }}=U_{\text {deformation }}-\pi r^{2} \gamma
$$

The top and bottom covers of the microfluidic reservoir are modelled as plates because their thickness is much smaller than the other dimensions. Similar to the microfluidic channels, the 
collapsed region is flat and for the top cover, deflection $w$ in the collapsed region $(\rho \leq r)$ is constant $-\delta$ to be determined. The deflection in the uncollapsed region satisfies

$$
\left(\frac{\mathrm{d}^{2}}{\mathrm{~d} \rho^{2}}+\frac{1}{\rho} \frac{\mathrm{d}}{\mathrm{d} \rho}\right)\left(\frac{\mathrm{d}^{2} w}{\mathrm{~d} \rho^{2}}+\frac{1}{\rho} \frac{\mathrm{d} w}{\mathrm{~d} \rho}\right)=0,(r<\rho \leq R)
$$

with boundary conditions

$$
\left\{\begin{array}{l}
\left.w\right|_{\rho=R}=0 \\
\left.\frac{\mathrm{d} w}{\mathrm{~d} x}\right|_{\rho=R}=0 \\
\left.w\right|_{\rho=r}=-\delta \\
\left.\frac{\mathrm{d} w}{\mathrm{~d} x}\right|_{\rho=r}=0
\end{array} .\right.
$$

The solution is

$$
=\left\{\begin{array}{cc}
w(\rho) & \rho \leq r \\
-\delta \frac{\left(R^{2}-r^{2}\right)\left(R^{2}-\rho^{2}\right)+2 R^{2} r^{2}\left[\frac{\rho^{2}}{r^{2}} \ln \frac{\rho}{R}+\frac{\rho^{2}}{R^{2}} \ln \frac{r}{\rho}-\left(1+2 \ln \frac{\rho}{R}\right) \ln \frac{r}{R}\right]}{\left(R^{2}-r^{2}\right)^{2}-4 R^{2} r^{2}\left[\ln \frac{r}{R}\right]^{2}} & r<\rho \leq R^{\prime}
\end{array}\right.
$$

and the deformation energy $U_{\text {deformation }}^{\text {top cover }}$ is then obtained as

$$
\begin{aligned}
U_{\text {deformation }}^{\text {top cove }} & =\int_{r}^{R} \frac{D_{\text {top }}}{2}\left[\left(\frac{\mathrm{d}^{2} w}{\mathrm{~d} \rho^{2}}+\frac{1}{\rho} \frac{\mathrm{d} w}{\mathrm{~d} \rho}\right)^{2}-2(1-v) \frac{\mathrm{d}^{2} w}{\mathrm{~d} \rho^{2}}\left(\frac{1}{\rho} \frac{\mathrm{d} w}{\mathrm{~d} \rho}\right)\right] 2 \pi \rho \mathrm{d} \rho \\
& =\frac{8 \pi D_{\text {top }} \delta^{2}\left(R^{2}-r^{2}\right)}{\left(R^{2}-r^{2}\right)^{2}-4 R^{2} r^{2}\left(\ln \frac{r}{R}\right)^{2}} .
\end{aligned}
$$

Similarly, we have

$$
U_{\text {deformation }}^{\text {bottom cover }}=\frac{8 \pi D_{\text {bottom }}(2 h-\delta)^{2}\left(R^{2}-r^{2}\right)}{\left(R^{2}-r^{2}\right)^{2}-4 R^{2} r^{2}\left(\ln \frac{r}{R}\right)^{2}} .
$$

Substitution of Eq. 18 and 19 into Eq. 14 gives the total potential energy as 


$$
U_{\text {total }}=\frac{8 \pi D_{\text {top }} \delta^{2}\left(R^{2}-r^{2}\right)}{\left(R^{2}-r^{2}\right)^{2}-4 R^{2} r^{2}\left(\ln \frac{r}{R}\right)^{2}}+\frac{8 \pi D_{\text {bottom }}(2 h-\delta)^{2}\left(R^{2}-r^{2}\right)}{\left(R^{2}-r^{2}\right)^{2}-4 R^{2} r^{2}\left(\ln \frac{r}{R}\right)^{2}}-\pi r^{2} \gamma .
$$

Minimization of total energy with respect to the deflection $\delta\left(\partial U_{\text {total }} / \partial \delta=0\right)$ gives exactly the same result as Eq. 8 because for both microfluidic channels and reservoirs we have the relations $U_{\text {deformation }}^{\text {top cover }} \propto D_{\text {top }} \delta^{2}$ and $U_{\text {deformation }}^{\text {bottom cover }} \propto D_{\text {bottom }}(2 h-\delta)^{2}$. Substitution of the calculated $\delta$ into Eq. 20 updates $U_{\text {total }}$ with only collapse radius $r$ to be determined.

$$
U_{\text {total }}=\frac{16 \pi D_{\text {eff }} h^{2}}{R^{2}}\left\{\frac{1-\left(\frac{r}{R}\right)^{2}}{\left[1-\left(\frac{r}{R}\right)^{2}\right]^{2}-4\left(\frac{r}{R}\right)^{2}\left(\ln \frac{r}{R}\right)^{2}}-\frac{\gamma R^{4}}{16 D_{\text {eff }} h^{2}}\left(\frac{r}{R}\right)^{2}\right\},
$$

where $D_{\text {eff }}$ has the same definition as in Eq. 10 .

Its normalization $U_{\text {total }} R^{2} /\left(D_{\text {eff }} h^{2}\right)$ depends only on the normalized collapse radius $r / R$ and normalized work of adhesion

$$
\bar{\gamma}_{\text {reservoir }}=\frac{\gamma R^{4}}{D_{\text {eff }} h^{2}}=\frac{\gamma\left(D_{\text {bottom }}+D_{\text {top }}\right) R^{4}}{2 D_{\text {top }} D_{\text {bottom }} h^{2}} .
$$

Figure 4 shows $U_{\text {total }} R^{2} /\left(D_{\text {eff }} h^{2}\right)$ versus $r / R$ for $\bar{\gamma}_{\text {reservoir }}=0,160,314.59,495.35$ and 800 . The collapse length $2 r_{\text {critical }}$, determined by minimizing the total potential energy $\left(\partial U_{\text {total }} / \partial r=\right.$ 0 and $\partial^{2} U_{\text {total }} / \partial r^{2}>0$ ), is also shown. Its solution only exists for $\bar{\gamma}_{\text {reservoir }}>314.59$, and is governed by

$$
\frac{\left(\frac{r_{\text {critical }}}{R}\right)^{2}-1-2 \ln \frac{r_{\text {critical }}}{R}}{\left[1-\left(\frac{r_{\text {critical }}}{R}\right)^{2}\right]^{2}-4\left(\frac{r_{\text {critical }}}{R}\right)^{2}\left(\ln \frac{r_{\text {critical }}}{R}\right)^{2}}=\frac{\sqrt{\bar{\gamma}_{\text {reservoir }}}}{4} .
$$

Substitution of $r_{\text {critical }} / R$ into Eq. 21 gives the minimum total potential energy $U_{\text {total. }}^{\min }$ For intermediate adhesion $314.59<\bar{\gamma}_{\text {reservoir }} \leq 495.35, U_{\text {total }}^{\min }$ is positive such that the collapse is meta stable. For strong adhesion $\bar{\gamma}_{\text {reservoir }}>495.35, U_{\text {total }}^{\min }$ is negative to give stable collapse. 
The critical values of normalized work of adhesion (weak-vs-intermediate, intermediate-vsstrong) for collapse of reservoirs are higher than the critical values of channels. This implies that, for the same feature size of microfluidic channels and reservoirs (e.g., $2 a=2 R$, same height $h$ and same effective bending stiffness $\left.D_{\text {eff }}\right)$, the microfluidic channel is easier to collapse than the reservoir.

\section{Comparison with Experiments}

Figure 5 shows the results from the collapse of recently reported, epidermal sweat monitoring devices [9]. The devices in Fig. 5 a-c have the same geometry but are made of PDMS with different mixture ratios (10:1, 30:1 and 50:1) and thus different Young's moduli (2.99MPa, $232 \mathrm{kPa}$ and $23.7 \mathrm{kPa}[17])$. However, the work of adhesion varies very little for these different mixture ratios $(10: 1,30: 1$ and $50: 1)$, from 37.9 to $41.9 \mathrm{~mJ} / \mathrm{m}^{2}$ [17], and is taken as a representative value $40 \mathrm{~mJ} / \mathrm{m}^{2}$ in the present study. The microfluidic system consists of both serpentine channels and circular reservoirs, to be compared with the models in sections 2 and 3 , respectively. The top and bottom cover thickness $t=0.1 \mathrm{~mm}$ and the height $2 \mathrm{~h}=0.3 \mathrm{~mm}$ for both microfluidic channels and reservoirs. The radius of the reservoir is $R=2 \mathrm{~mm}$ and the width of the channel is $2 a=1 \mathrm{~mm}$.

Table 1 shows the normalized work of adhesion for the three devices, each with the microfluidic channels and reservoirs. For 10:1 PDMS (highest modulus 2.99MPa), $\bar{\gamma}_{\text {channel }}<3.0$ and $\bar{\gamma}_{\text {reservoir }}<314.59$ (i.e., weak adhesion for both channels and reservoirs) such that no collapse occurs. For 30:1 PDMS (intermediate modulus $232 \mathrm{kPa}$ ), $\bar{\gamma}_{\text {channel }}<36$ (weak adhesion) while $\bar{\gamma}_{\text {reservoir }}>495.35$ (strong adhesion) collapse occurs in circular reservoirs but not in channels. For 50:1 PDMS (lowest modulus $23.7 \mathrm{kPa}$ ), $36<\bar{\gamma}_{\text {channel }}<113.78$ (i.e., intermediate 
adhesion) and $\bar{\gamma}_{\text {reservoir }}>495.35$ (i.e., strong adhesion), the circular reservoirs collapse and the channels may collapse too. As shown in Table 1, the experimental results agree well with the analytical models.

\section{Conclusion}

Analytical models are established in this paper for self-collapse of microfluidic channels and reservoirs in soft, epidermal microfluidic devices. Three different collapse states, namely no collapse, meta stable collapse and stable collapse, exist, and they are controlled by a single parameter, the normalized work of adhesion, which combines the effects of channel/reservoir geometry, work of adhesion and bending stiffness of top and bottom covers of channel/reservoir. The study provides design guidelines to avoid the problem of self-collapse in epidermal microfluidic devices.

\section{Acknowledgements}

X.F. acknowledge the support from the National Basic Research Program of China (Grant No. 2015CB351900) and National Natural Science Foundation of China (Grant No. 11320101001). Y.H. acknowledges the support from the NSF (Grant No. DMR1121262, CMMI1300846, CMMI1400169 and CMMI1534120) and the NIH (Grant No. R01EB019337).

\section{Appendix A. Proof of Flatness of Collapsed Region}

For microfluidic channel, if the collapsed region is non-flat, a rotation angle $\theta$ exists at the boundary of collapsed region and uncollapsed region $(|x|=b)$, as showed in Fig. A1. Here we prove that in energy minimum state $\theta=0$ and thus the collapsed region is indeed flat. 
For simplicity, we only consider the region $x>0$ due to symmetry for $x<0$ region. The deflection at $x=b$ is assumed $-\delta$ and the rotation is $\theta$. The uncollapsed region of top cover satisfies

$$
\frac{\mathrm{d}^{4} w}{\mathrm{~d} x^{4}}=0 \quad(b<x<a)
$$

with the boundary conditions

$$
\left\{\begin{array}{l}
\left.w\right|_{x=a}=0 \\
\left.\frac{\mathrm{d} w}{\mathrm{~d} x}\right|_{x=a}=0 \\
\left.w\right|_{x=b}=-\delta \\
\left.\frac{\mathrm{d} w}{\mathrm{~d} x}\right|_{x=b}=\theta
\end{array}\right.
$$

Its solution is

$$
w=\frac{(a-x)^{2}\left[3 b \delta-2 x \delta+b^{2} \theta-b x \theta+a(-\delta-b \theta+x \theta)\right]}{(a-b)^{3}} .
$$

The bending moment at $x=b$,

$$
M_{\text {top }}=\left.D_{\text {top }} \frac{\mathrm{d}^{2} w}{\mathrm{~d} x^{2}}\right|_{x=b}=\frac{2 D_{\text {top }}[3 \delta-2(a-b) \theta]}{(a-b)^{2}} .
$$

The deformation energy is

$$
U_{\text {deformation }}^{\text {top free }}=\int_{b}^{a} \frac{D_{\text {top }}}{2}\left(\frac{\mathrm{d}^{2} w}{\mathrm{~d} x^{2}}\right)^{2} \mathrm{~d} x=\frac{2 D_{\text {top }}\left[3 \delta^{2}-3(a-b) \delta \theta+(a-b)^{2} \theta^{2}\right]}{(a-b)^{3}}
$$

Similarly, the uncollapsed region of bottom cover also satisfies Eq. A1 but with slightly different boundary conditions,

$$
\left\{\begin{array}{c}
\left.w\right|_{x=a}=0 \\
\left.\frac{\mathrm{d} w}{\mathrm{~d} x}\right|_{x=a}=0 \\
\left.w\right|_{x=b}=2 h-\delta \\
\left.\frac{\mathrm{d} w}{\mathrm{~d} x}\right|_{x=b}=\theta
\end{array}\right.
$$

Its solution is 


$$
w=\frac{(a-x)^{2}\left[-6 b h+3 b \delta+4 h x-2 x \delta+b^{2} \theta-b x \theta+a(2 h-\delta-b \theta+x \theta)\right]}{(a-b)^{3}} .
$$

The bending moment at $x=b$,

$$
M_{\text {bottom }}=\left.D_{\text {bottom }} \frac{\mathrm{d}^{2} w}{\mathrm{~d} x^{2}}\right|_{x=b}=\frac{2 D_{\text {top }}[3 \delta-6 h-2(a-b) \theta]}{(a-b)^{2}} .
$$

The deformation energy is given by

$$
\begin{aligned}
U_{\text {deformation }}^{\text {bottom free }} & =\int_{b}^{a} \frac{D_{\text {bottom }}}{2}\left(\frac{\mathrm{d}^{2} w}{\mathrm{~d} x^{2}}\right)^{2} \mathrm{~d} x \\
& =\frac{2 D_{\text {bottom }}\left[3(2 h-\delta)^{2}+3(a-b)(2 h-\delta) \theta+(a-b)^{2} \theta^{2}\right]}{(a-b)^{3}} .
\end{aligned}
$$

The collapsed region is composed of both top and bottom covers (assume its bending stiffness is $D_{\text {composite }}$ ) and is subjected to bending moment $M_{\text {top }}+M_{\text {bottom. Thus it satisfies }}$

$$
\frac{M_{\text {top }}+M_{\text {bottom }}}{D_{\text {composite }}}=\frac{\theta}{b} .
$$

Combining Eq. A4, A8 and $\mathrm{A} 10$ gives

$$
\theta=\frac{6 b\left[D_{\text {top }} \delta-D_{\text {bottom }}(2 h-\delta)\right]}{(a-b)\left[a+b\left(4 D_{\text {bottom }}-D_{\text {composite }}+4 D_{\text {top }}\right)\right]}
$$

The deformation energy in the collapsed region is

$$
U_{\text {deformation }}^{\text {collapsed region }}=b \frac{\left(M_{\mathrm{top}}+M_{\mathrm{bottom}}\right)^{2}}{2 D_{\text {composite }}} .
$$

The total deformation energy $U_{\text {deformation }}$ is the sum of $U_{\text {deformation }}^{\text {top free }} U_{\text {deformation }}^{\text {bottom free }}$ and $U_{\text {deformation }}^{\text {colllapsed region }}$ with substitution of $\theta$ using Eq. A11, which has complex expression but can be written as

$$
U_{\text {deformation }}=\Phi\left(a, b, D_{\text {top }}, D_{\text {bottom }}, D_{\text {composite }}\right)\left[D_{\text {top }} \delta^{2}+D_{\text {bottom }}(2 h-\delta)^{2}\right]
$$


In energy minimum state $\delta$ is determined from $\partial U_{\text {deformation }} / \partial \delta=0$, and despite the complex

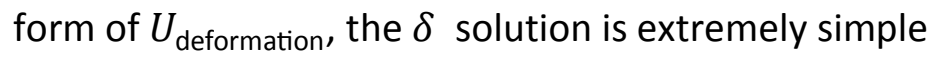

$$
\frac{\delta}{2 h}=\frac{D_{\text {bottom }}}{D_{\text {top }}+D_{\text {bottom }}}
$$

Substitution of Eq. A14 into Eq. A11 gives $\theta=0$, which shows in energy minimum state, the collapsed region is always flat.

\section{References}

[1] Patel, S., Park, H., Bonato, P., Chan, L., and Rodgers, M., 2012, "A review of wearable sensors and systems with application in rehabilitation," J Neuroeng Rehabil, 9.

[2] Bonato, P., 2010, "Wearable Sensors and Systems From Enabling Technology to Clinical Applications," leee Eng Med Biol, 29(3), pp. 25-36.

[3] Webb, R. C., Bonifas, A. P., Behnaz, A., Zhang, Y. H., Yu, K. J., Cheng, H. Y., Shi, M. X., Bian, Z. G., Liu, Z. J., Kim, Y. S., Yeo, W. H., Park, J. S., Song, J. Z., Li, Y. H., Huang, Y. G., Gorbach, A. M., and Rogers, J. A., 2013, "Ultrathin conformal devices for precise and continuous thermal characterization of human skin," Nat Mater, 12(10), pp. 938-944.

[4] Dagdeviren, C., Shi, Y., Joe, P., Ghaffari, R., Balooch, G., Usgaonkar, K., Gur, O., Tran, P. L., Crosby, J. R., Meyer, M., Su, Y. W., Webb, R. C., Tedesco, A. S., Slepian, M. J., Huang, Y. G., and Rogers, J. A., 2015, "Conformal piezoelectric systems for clinical and experimental characterization of soft tissue biomechanics," Nat Mater, 14(7), pp. 728-+.

[5] Zhang, Y. H., Webb, R. C., Luo, H. Y., Xue, Y. G., Kurniawan, J., Cho, N. H., Krishnan, S., Li, Y. H., Huang, Y. G., and Rogers, J. A., 2016, "Theoretical and Experimental Studies of Epidermal Heat Flux Sensors for Measurements of Core Body Temperature," Adv Healthc Mater, 5(1), pp. 119127.

[6] Matzeu, G., Florea, L., and Diamond, D., 2015, "Advances in wearable chemical sensor design for monitoring biological fluids," Sensor Actuat B-chem, 211, pp. 403-418.

[7] Salvo, P., Di Francesco, F., Costanzo, D., Ferrari, C., Trivella, M. G., and De Rossi, D., 2010, "A Wearable Sensor for Measuring Sweat Rate," leee Sensors Journal, 10(10), pp. 1557-1558.

[8] Bandodkar, A. J., and Wang, J., 2014, "Non-invasive wearable electrochemical sensors: a review," Trends Biotechnol, 32(7), pp. 363-371.

[9] Kang, D., Koh, A., Xue, Y., Lee, S., Pielak, R. M., Kim, J., Hwang, T., Min, S., Banks, A., Bastien, P., Manco, M. C., Wang, L., R., A. K., Jang, K., Won, P., Han, S., Paik, U., Slepian, M. J., Balooch, G., Huang, Y., and Rogers, J. A., 2016, "Skin-Like, Wearable Microfluidic Systems Capable of Capture, Storage and Colorimetric Sensing of Sweat," Sci Transl Med.

[10] Kim, D. H., Lu, N. S., Ma, R., Kim, Y. S., Kim, R. H., Wang, S. D., Wu, J., Won, S. M., Tao, H., Islam, A., Yu, K. J., Kim, T. I., Chowdhury, R., Ying, M., Xu, L. Z., Li, M., Chung, H. J., Keum, H., McCormick, M., Liu, P., Zhang, Y. W., Omenetto, F. G., Huang, Y. G., Coleman, T., and Rogers, J. A., 2011, "Epidermal Electronics," Science, 333(6044), pp. 838-843. 
[11] Zhang, Y. H., Xu, S., Fu, H. R., Lee, J., Su, J., Hwang, K. C., Rogers, J. A., and Huang, Y. G., 2013, "Buckling in serpentine microstructures and applications in elastomer-supported ultrastretchable electronics with high areal coverage," Soft Matter, 9(33), pp. 8062-8070.

[12] Huang, Y. G. Y., Zhou, W. X., Hsia, K. J., Menard, E., Park, J. U., Rogers, J. A., and Alleyne, A. G., 2005, "Stamp collapse in soft lithography," Langmuir, 21(17), pp. 8058-8068.

[13] Xue, Y. G., Zhang, Y. H., Feng, X., Kim, S., Rogers, J. A., and Huang, Y. G., 2015, "A theoretical model of reversible adhesion in shape memory surface relief structures and its application in transfer printing," J Mech Phys Solids, 77, pp. 27-42.

[14] Mastrangelo, C. H., and Hsu, C. H., "A simple experimental technique for the measurement of the work of adhesion of microstructures," IEEE, pp. 208-212.

[15] Mastrangelo, C. H., and Hsu, C., 1993, "Mechanical stability and adhesion of microstructures under capillary forces. I. Basic theory," J Microelectromech S, 2(1), pp. 33-43.

[16] de Boer, M. P., and Michalske, T. A., 1999, "Accurate method for determining adhesion of cantilever beams," J Appl Phys, 86(2), pp. 817-827.

[17] Yu, Y. L., Sanchez, D., and Lu, N. S., 2015, "Work of adhesion/separation between soft elastomers of different mixing ratios," J Mater Res, 30(18), pp. 2702-2712. 


\section{Figures}

Figure 1. Schematic diagrams for (a) the microfluidic channel and (b) its collapsed state.

Figure 2. Normalized total potential energy versus normalized collapse length of the microfluidic channel for several values of normalized worked adhesion. The red stars mark the critical collapse length $b_{\text {critical }} / a$ that minimizes the total potential energy.

Figure 3. Schematic diagram of (a) the microfluidic reservoir and (b) its collapsed state.

Figure 4. Normalized total potential energy versus normalized collapse radius of the microfluidic reservoir for several values of normalized worked adhesion. The red stars mark the critical collapse radius $r_{\text {critical }} / R$ that minimizes the total potential energy.

Figure 5. Analytic and experimental results of microfluidic channel/reservoir collapse for three devices made of PDMS of different mixture ratios. The collapsed regions are marked with red circles.

Figure A1. Cross section of (a) the microfluidic channel and (b) its collapsed state with non-flat collapsed region. A rotation angle $\theta$ exists at position $x=b$.

\section{Tables}

Table 1. Calculated normalized work of adhesion for the three devices with comparison of analytical models and experimental results. 
(a)

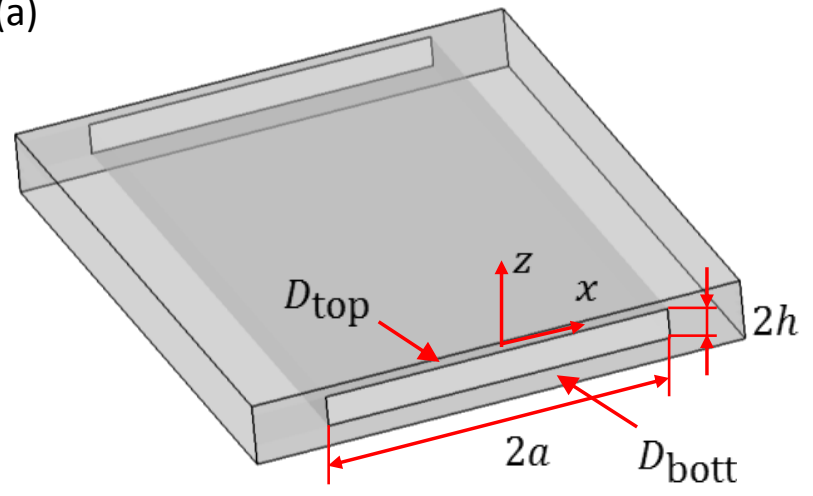

(b)

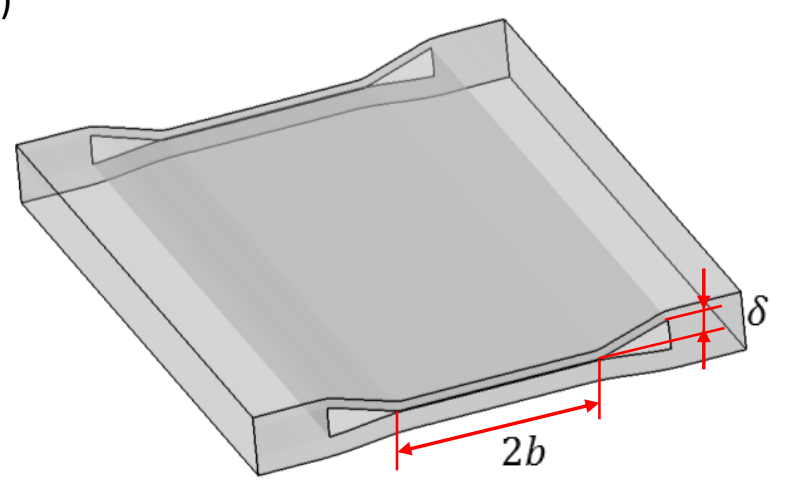

Figure 1 


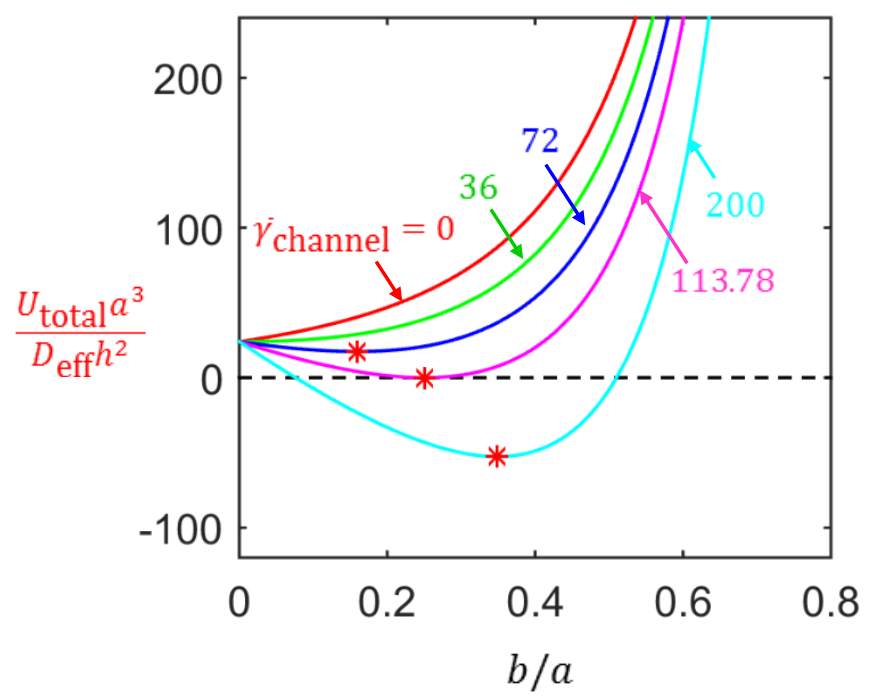

Figure 2 
(a)

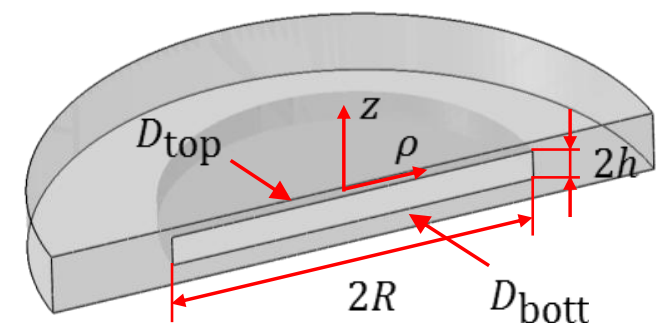

(b)

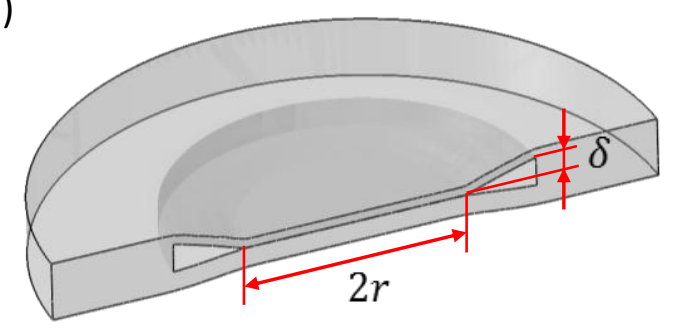

Figure 3 


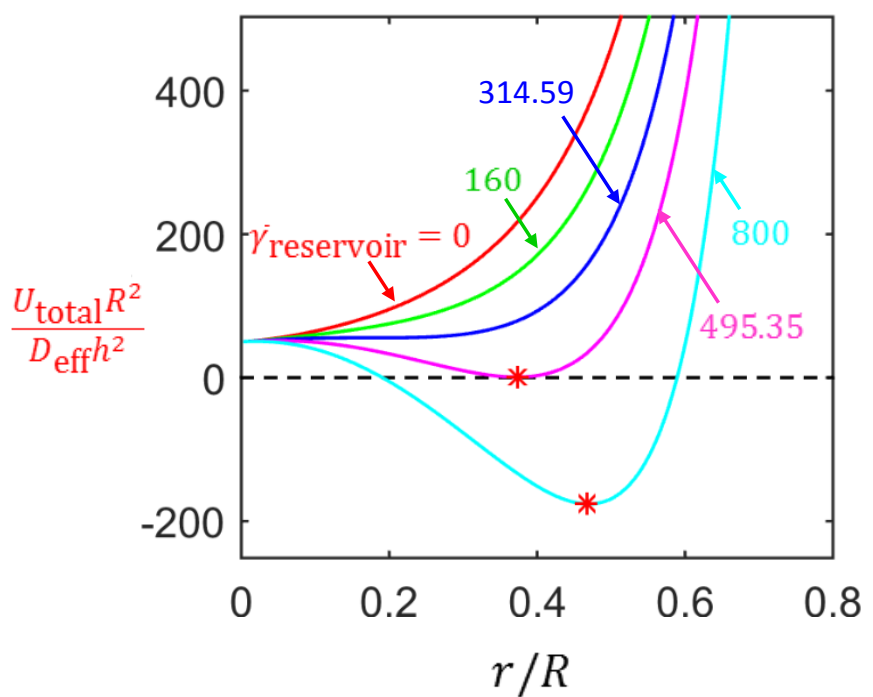

Figure 4 
(a)

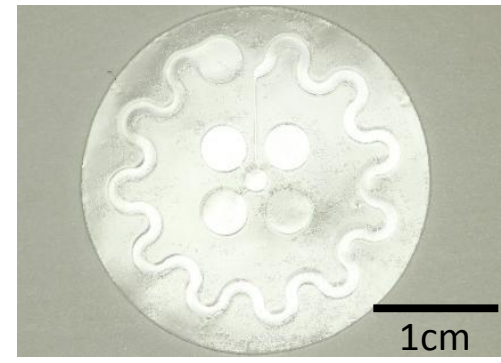

10:1 PDMS

( $\mathrm{E}=2.99 \mathrm{Mpa})$ (b)

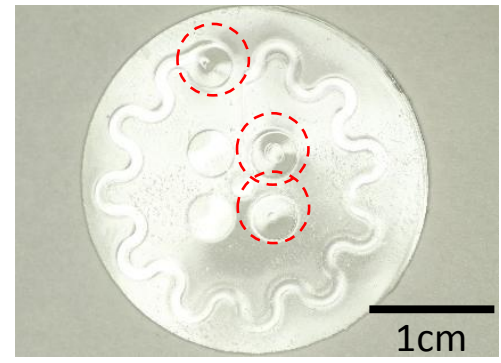

30:1 PDMS

$(\mathrm{E}=232 \mathrm{kPa})$

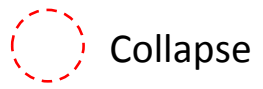

(c)

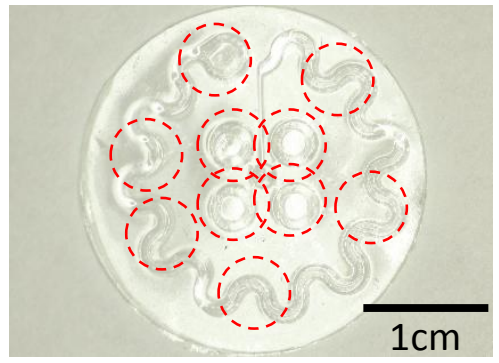

50:1 PDMS

$(E=23.7 \mathrm{kPa})$

Figure 5 


\begin{tabular}{lccc}
\hline & $\begin{array}{c}\text { Normalized Work of } \\
\text { Adhesion }\end{array}$ & $\begin{array}{c}\text { Analytical } \\
\text { Model }\end{array}$ & $\begin{array}{c}\text { Experimental } \\
\text { Result }\end{array}$ \\
10:1 PDMS, channel & $\bar{\gamma}_{\text {channel }}=0.33$ & No Collapse & No Collapse \\
30:1 PDMS, channel & $\bar{\gamma}_{\text {Channel }}=42.2$ & No Collapse & No Collapse \\
50:1 PDMS, channel & $\bar{\gamma}_{\text {channel }}=52.6$ & Meta-stable Collapse & Collapse \\
10:1 PDMS, reservoir & $\bar{\gamma}_{\text {reservolr }}=85.6$ & No Collapse & No Collapse \\
30:1 PDMS, reservoir & $\bar{\gamma}_{\text {reservoir }}=1103.4$ & Stable Collapse & Collapse \\
50:1 PDMS, reservoir & $\bar{\gamma}_{\text {reservoir }}=10802$ & Stable Collapse & \\
\hline
\end{tabular}


(a)
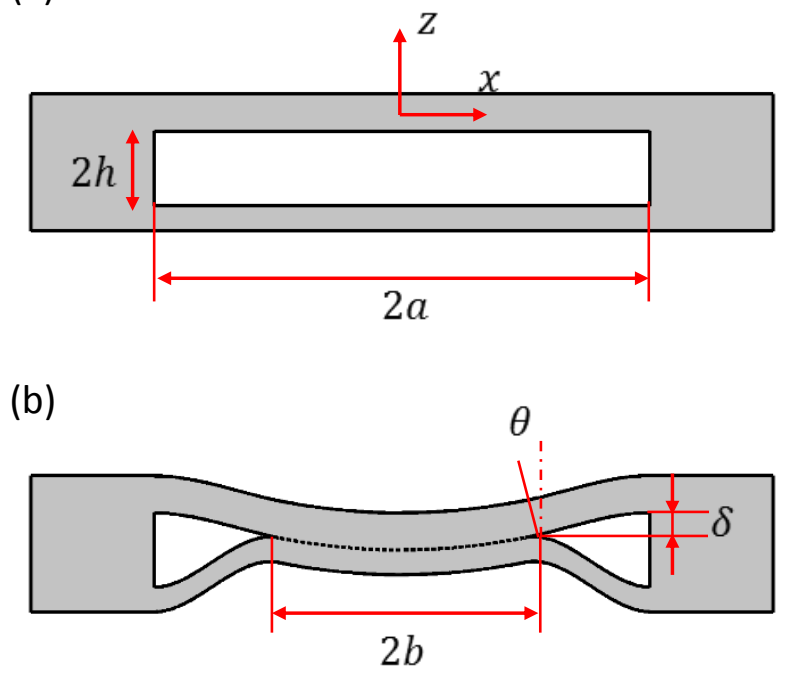

Figure A1 